\title{
A tool for IFC building energy performance simulation suitability checking.
}

\author{
G.N. Lilis, G. Giannakis \& K. Katsigarakis \\ Department of Production Engineering and Management, Technical University of Crete, Chania, Greece \\ D.V. Rovas \\ Institute for Environmental Design and Engineering, University College London, London, UK
}

\begin{abstract}
Data quality of BIM models is a key determinant in the value that can be extracted out of these data. Yet, despite this importance the discussion of data quality is often relegated to an afterthought. One potential use of BIM model data is the generation of building energy performance simulation models. Within this paper a checking procedure is presented, to ensure that user-supplied BIM models meet threshold data quality criteria and are suitable for the generation of input data files for energy analysis. The checking procedure comprises of three sets of checking operations: consistency, correctness and completeness checks. Consistency checks ensure that the input data are schema compatible; data completeness checks invoke the sequential execution of checking rules to verify the existence of required data; data correctness checks perform more elaborate detection of geometric errors appearing in surfaces, space volumes and clashes between architectural elements, which affect the building energy performance simulation model generation process. The checking procedure has been implemented and tested in two case-study buildings. Although the BIM modelers had been provided with modeling guidelines, multiple inaccuracies and data insufficiencies were still present, highlighting the importance of a posteriori process implementation that checks the validity of the model in relation to the purpose of its use.
\end{abstract}

\section{INTRODUCTION}

BIM models are becoming increasingly available for new but also for existing buildings. Similar to modeling in other domains, there is always the question of what should be modeled and at what level of detail. The level of modeling detail is intricately linked to the intended use of these models for various purposes that might include architectural and building services design, sustainability assessment, and facility management to name but a few. Capturing data requirements is an important first step, followed by the need to ensure that the data provided are of sufficient quality to be useful for the intended purpose. If such purpose is the setting up of Building Energy Performance Simulation (BEPS) models, often required in the context of sustainability discussions, data integration from multiple domains is often required (Cormier et al., 2011; Gudnason et al., 2014; Senave and Boeykens, 2015). To yield any useful outcomes, the input data provided in the first instance should meet certain quality threshold criteria (Gholami et al., 2015). However, due to high-complexity of data required for energy analysis purposes, it is quite common that the provided data fail to meet such criteria (Hitchcock and Wong, 2011; Nasyrov et al., 2014). Quality control in that sense becomes as important as the modeling process itself, while having ways to capture such errors is quite important, given the complexity of the problem. In this paper, data quality checking operations are classified into three categories, which are implemented in a sequential order and include consistency, completeness and correctness checking operations, mirroring checking and ensuring interoperability at three levels: syntactic, semantic and pragmatic.

For consistency checks, the syntactic checking of the input file is performed to ensure that the provided BIM model adheres to a specific schema. For the purposes of this paper BIM files are assumed to follow the IFC4 Add2 schema (ISO16739, 2013). In the completeness, checks the data integrity of the BIM files is investigated, using a number of checking rules and any missing information is reported. These checking rules are implemented using the IFC Model View Definition (MVD) methodology. Recently, several MVDs for BEPS model generation have been proposed (Pinheiro et al., 2018; Alshehril et al., 2017). Finally in the correctness checks, the BIM files' information is checked for possible errors. These checking operations should be applied to any BIM input data file in order to guarantee correctness of generated BEPS models.

These operations are included in a stand-alone BIM for BEPS suitability checking tool, which is the main topic of the present work. As it is illustrated by the diagram of figure 1 , the tool receives IFC4 BIM files, designed followed specific guidelines and exported via a dedicated exporter, passes them trough the three data quality checking stages (consistency, completeness and correctness) and reports the data quality results in its output. The tool is an essential first step in the process of generation 
input data files for the EnergyPlus simulation environment.

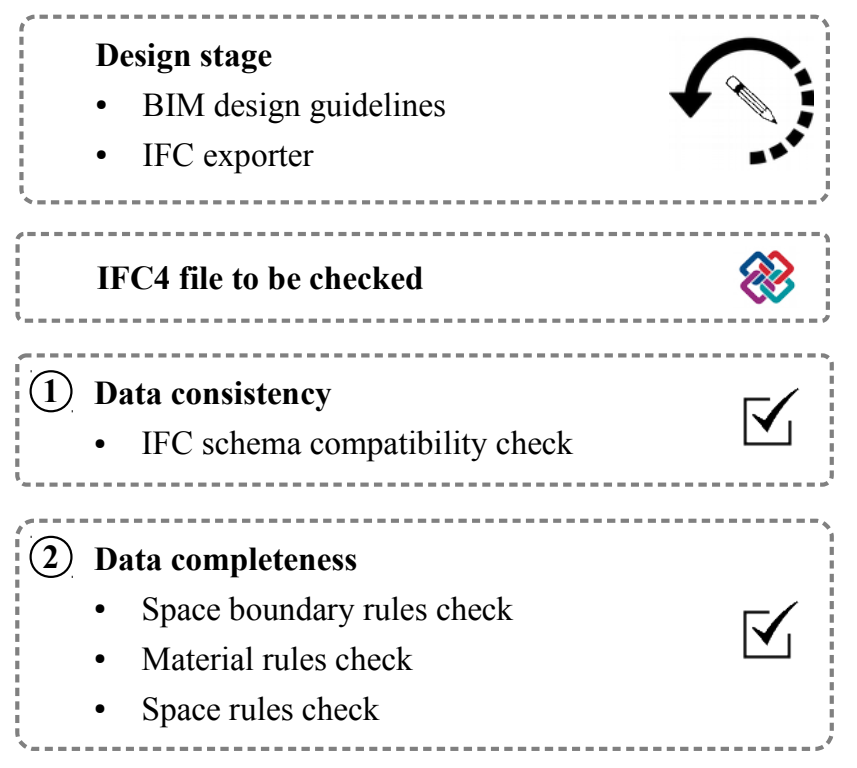

(3) Data correctness

- Geometric Error Detection

Checking results report

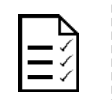

Figure 1: Overview of the proposed BIM data quality checking process.

\section{DATA QUALITY}

As described in the introduction, data quality checking operations can be classified into three categories: data consistency, data correctness and data completeness checks as described in the following sections.

\subsection{Data Consistency}

Building Information Models, being an objectbased digital representation of a building, are an information-rich source for setting up BEPS models. More specifically, in the current version of IFC (IFC4 Add2), which became an ISO standard (ISO16739, 2013), the data are stored in a STEP file, with the schema defined using the EXPRESS data modeling language. The IFC file which is usually generated by the exporter component of a BIMauthoring tool, should be consistent with the EXPRESS schema. This consistency check of the input IFC precedes any other completeness or correctness check.

\subsection{Data Completeness}

BEPS model generation requires certain data to be present in the input IFC BIM files and to be "BEPS- complete". To ensure that IFC files contain these data a dedicated IFC exporter, a document containing appropriate BIM design guidelines and a BIM checking tool have been developed and offered to the user of the platform, which are detailed in the following sections.

\subsubsection{IFC Exporter}

Many popular commercial BIM authoring tools e.g. Revit $^{\mathrm{TM}}$ (Autodesk, 2018), ArchiCad ${ }^{\mathrm{TM}}$ (Graphisoft, 2018) and ALLPLAN ${ }^{\mathrm{TM}}$ (Nemetschek Group, 2018), support exportation of IFC files. However, exportation is often not perfect: the exported models can be of poor quality and not directly usable. The Revit ${ }^{\mathrm{TM}}$ IFC4 DTV exporter is far from perfect: although, IFC can incorporate information about thermal and optical properties of each building entitys construction material, internal gains (schedules and densities) and inverse relations in case of curtain walls, the current version of the exporter is not capable of exporting this information. Therefore, a dedicated Revit based IFC exporter has been developed which supports these data exportation needs.

\subsubsection{BIM design guidelines}

BIM models exported by appropriate exporters are not always suitable for BEPS model generation as information might be missing or their data might be incorrectly defined. For example, BIM building geometric data are oftentimes inappropriate for BEMS model generation. For these reason BIM design guidelines have been published (Maile et al., 2013) in order to guide the designers towards building designs suitable for BEPS model generation. Aligned to this direction the tools allows the user to download a pdf document containing appropriate guidelines. This document contains geometric as well as non-geometric rules to export a suitable ("BEPS-complete" and "BEPS-correct") IFC4 file. Snapshots of this document are displayed in figure 2.

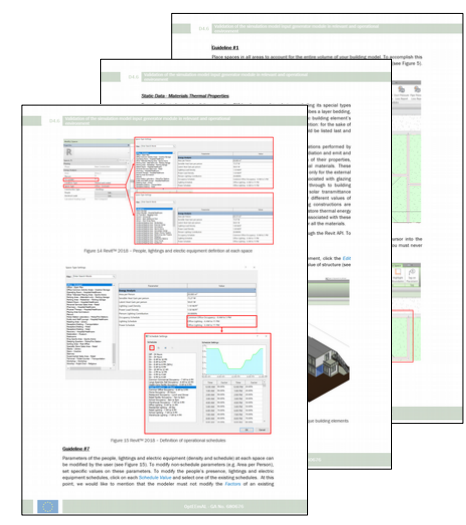

Figure 2: Snapshots of BIM design guidelines. 


\subsubsection{BIM Checking tool}

In this section checking rules, embedded in the BIM Checking tool, are presented; these rules are applied to check the data availability in the IFC4 file, from a Building Energy Performance Simulation (BEPS) perspective. Three main categories of rules are considered, described thoroughly below: space boundary rules; space rules; and material rules.

\section{Space boundary rules}

From a BEPS viewpoint, the partition of building construction (interior/exterior walls, floors, roofs) and opening (door, window) surfaces into $2^{\text {nd }}$-level space boundary (Bazjanac, 2010) surfaces, is a prerequisite. This process is performed by the Common Boundary Intersection Projection (CBIP) tool. The result of the CBIP tools execution is the enrichment of the IFC file with relevant $2^{\text {nd }}$-level space boundary information (IfcRelSpaceBoundary2ndLevel class population).

Before checking the $2^{n d}$-level space boundary content of the IFC file certain data requirements of the CBIP $2^{\text {nd }}$-level space boundary calculation and IFC data enrichment process is checked by the BIM Checking tool. More specifically, the existence of the following three IFC objects is examined: (a) one building object; (b) one site object attached to the building (to define the ground boundary conditions of the BEPS); (c) at least one space object (to obtain the space boundary surfaces related to this space volume) (d) at least on building element object (wall, slab, etc); and some unit objects.

The $2^{\text {nd }}$-level space boundary content of the enriched IFC file is checked by the BIM checking tool, using the following four checking rules, which are implemented sequentially:

1. The existence of the $2^{\text {nd }}$-level space boundaries is checked by examining the presence of the IfcRelSpaceBoundary2ndLevel class instances in the enriched IFC file. If there are no such instances, the following error message is reported: "2nd level space boundaries are missing". If this rule is passed the next rule is examined.

2. For each IfcRelSpaceBoundary2ndLevel populated instance, the presence of its related building element is examined. If related element exists the check proceeds to the next rule, otherwise a "related building element is missing" message, is reported.

3. For each IfcRelSpaceBoundary2ndLevel instance, the related building elements type, the value of the ExeternalOrInternal property, the corresponding space boundary (if the space boundary is INTERNAL) and the parent space boundary (if the space boundary refers to an opening), are examined. If any information is missing error messages are reported according to Table 3.

4. For each IfcRelSpaceBoundary2ndLevel instance the relating space index, is checked. If this index is missing, the following error message is reported: "relating space is missing".

5. Finally, the last rule checks if there is an IfcRelSpaceBoundary2ndLevel instance with ExeternalOrInternal property value "EXTERNAL_EARTH". If no such instance exists, a warming message "ground boundary condition is missing", is reported.

If any missing information is detected appropriate error messages are generated, which are summarized for different space boundary types (internal, external, corresponding and parent) and related building elements (wall, slab, door, window, plate, opening and virtual element) in table 1.

Table 1: Error messages of space boundary checking rules E:external, I:internal, C:corresponding, P:parent, M:missing

\begin{tabular}{|c|c|c|c|c|}
\hline IFC class & $\mathbf{E} / \mathbf{I}$ & $\mathbf{C}$ & $\mathbf{P}$ & Error message \\
\hline IfcWall & I & M & - & $\begin{array}{l}\text { corresponding space } \\
\text { boundary is missing for } \\
\text { internal wall }\end{array}$ \\
\hline IfcSlab & I & M & - & $\begin{array}{l}\text { corresponding space } \\
\text { boundary is missing for } \\
\text { internal slab }\end{array}$ \\
\hline \multirow{3}{*}{ IfcDoor } & $\mathrm{E}$ & - & M & $\begin{array}{l}\text { parent space boundary is } \\
\text { missing for external door }\end{array}$ \\
\hline & I & - & M & $\begin{array}{l}\text { parent space boundary is } \\
\text { missing for internal door }\end{array}$ \\
\hline & I & M & - & $\begin{array}{l}\text { corresponding space } \\
\text { boundary is missing for } \\
\text { internal door }\end{array}$ \\
\hline \multirow{3}{*}{ IfcWindow } & $\mathrm{E}$ & - & M & $\begin{array}{l}\text { parent space boundary is } \\
\text { missing for external window }\end{array}$ \\
\hline & I & - & M & $\begin{array}{l}\text { parent space boundary is } \\
\text { missing for internal window }\end{array}$ \\
\hline & I & M & - & $\begin{array}{l}\text { corresponding space } \\
\text { boundary is missing for } \\
\text { internal window }\end{array}$ \\
\hline \multirow{2}{*}{ IfcPlate } & $\mathrm{E}$ & - & M & $\begin{array}{l}\text { parent space boundary is } \\
\text { missing for plate }\end{array}$ \\
\hline & I & - & - & $\begin{array}{l}\text { plate cannot have an } \\
\text { internal space boundary }\end{array}$ \\
\hline IfcOpening & $\mathrm{E}$ & - & - & $\begin{array}{l}\text { external space boundary } \\
\text { with IfcOpeningElement as } \\
\text { related building element } \\
\text { was found }\end{array}$ \\
\hline $\begin{array}{l}\text { IfcVirtual } \\
\text { Element }\end{array}$ & $\mathrm{E}$ & - & - & $\begin{array}{l}\text { external space boundary } \\
\text { with IfcVirtualElement as } \\
\text { related building element } \\
\text { was found }\end{array}$ \\
\hline
\end{tabular}




\section{Space rules}

Some BEPS programs require information about the space occupants presence, artificial lighting, electrical equipment operation (which act as thermal sources in terms of BEPS) and related operation schedules. These data are supported by the provided IFC Revit ${ }^{\mathrm{TM}}$ exporter, which populates a new Pset class, named Pset_Space InternalGainsDesign, which is assigned to each IfcSpace class. The properties of this new class are checked and appropriate error messages are reported according to table 2 .

Table 2: Error messages of space checking rules

\begin{tabular}{|l|l|}
\hline Checked property & Error message \\
\hline OccupancySchedule & occupancy schedule is missing \\
\hline LightingSchedule & lighting schedule is missing \\
\hline EquipmentSchedule & equipment schedule is missing \\
\hline HeatGainLighting & $\begin{array}{l}\text { heat gain lighting parameter } \\
\text { is missing }\end{array}$ \\
\hline AreaPerOccupant & $\begin{array}{l}\text { area per occupant parameter } \\
\text { is missing }\end{array}$ \\
\hline HeatGainPerOccupant & $\begin{array}{l}\text { heat gain equipment parameter } \\
\text { is missing }\end{array}$ \\
\hline InfiltrationRate & $\begin{array}{l}\text { infiltration rate parameter } \\
\text { is missing }\end{array}$ \\
\hline
\end{tabular}

\section{Material rules}

BEPS require a number of properties to be assigned to materials of various building constructions. These properties, depending on the material type, are: for opaque materials Conductivity, Density and Specific Heat; for transparent materials Ufactor and Solar heat gain coefficient (SHGC). Two checking rules depending on the material type are applied, which report error messages that are listed in tables 3 (opaque materials) and 4 (transparent materials).

Table 3: Error messages of opaque material property checking

\begin{tabular}{|l|l|}
\hline Checked property & Error message \\
\hline SpecificHeatCapacity & $\begin{array}{l}\text { SpecificHeatCapacity parameter } \\
\text { is missing }\end{array}$ \\
\hline ThermalConductivity & $\begin{array}{l}\text { ThermalConductivity parameter } \\
\text { is missing }\end{array}$ \\
\hline MassDensity & MassDensity parameter is missing \\
\hline
\end{tabular}

Table 4: Error messages of transparent material property checking

\begin{tabular}{|l|l|}
\hline Checked property & Error message \\
\hline Visual light transmittance & $\begin{array}{l}\text { Visual light transmittance } \\
\text { parameter is missing }\end{array}$ \\
\hline solar heat gain coefficient & $\begin{array}{l}\text { solar heat gain coefficient } \\
\text { parameter is missing }\end{array}$ \\
\hline heat transfer coefficient & $\begin{array}{l}\text { heat transfer coefficient } \\
\text { parameter is missing }\end{array}$ \\
\hline thermal resistance & $\begin{array}{l}\text { thermal resistance parameter } \\
\text { is missing }\end{array}$ \\
\hline
\end{tabular}

\subsection{Data Correctness}

Even if the BEPS modeler uses an error-free exporter, has followed appropriate design guidelines to develop the BIM file and the exported IFC file has passed all data completeness checks, there are cases where the IFC data might be inaccurate. In these cases manual corrections should be applied. These inaccuracies of the values contained in IFC classes affect the quality of the generated BEPS models. There are various such inaccuracies. For example, an intersection or clash between the geometric description of an internal building volume and the geometric description of a surrounding building construction element such as an internal wall, affect the related space boundary surfaces (surfaces through which the space exchanges heat with its environment), and thus the correctness of the respective BEPS model is affected as well. In the present work only the geometric inaccuracies will be examined, however the data correctness checks can be extended to non-geometric elements, such as the properties of building elements which should obtain numerical values within a predefined range.

There are certain geometric inaccuracies which affect the generation of a building energy performance simulation input data file, grouped in the following three categories: clashes; surface errors; and space incorrect definitions Lilis et al. (2015). These geometric error types are presented in the following sections.

\subsubsection{Clashes}

Clashes are intersections between the solid geometric representations of architectural building components (walls, slabs, spaces, etc.). Certain clash types affect the generation of BEPS models by altering the space boundary surface topology of the building (Bazjanac, 2010). These clash types refer to intersections of the building internal space volumes with other neighbor solid geometric representations (walls, slabs, etc.), as displayed in the example of a special clash type (containment) in part II of figure 3 . In this example, the wall volume is contained entirely in the space volume resulting to omitting the space boundary surfaces related to this wall. All other (not space-related) intersections affect BEPS model generation only on when the intersection surfaces are attached to pairs of building space volumes or a building space volume and the building site. A Wall Opening - Wall Opening clash example, where the intersection volume is attached to a space pair, causing space boundary duplication, is illustrated in part III of figure 3 . 
I. Slab - Wall clash

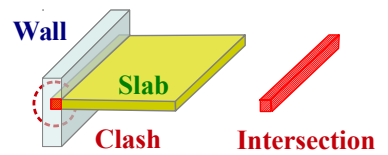

A building slab intersects with a building wall. This clash type does not affect BEP simulation model generation because the intersection volume is not attached to other building space or site volumes.
II. Space - Wall clash

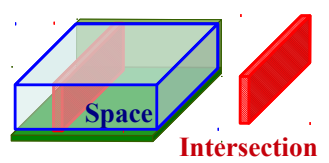

A building space contains an internal wall. This clash type affects BEP simulation model generation because the wall space boundary surfaces are omitted.
III. Wall Opening - Wall Opening clash

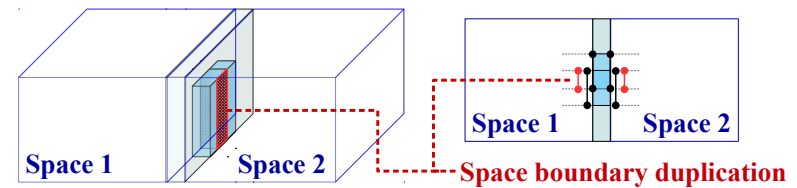

A wall opening intersects with another wall opening. This non-space clash affects BEP simulation model generation because the intersection surfaces are attached to a space volume, resulting to a space boundary duplication.

Figure 3: Clash types and their effect to BEPS model generation.

\subsubsection{Surface Errors}

Surface errors exist, when one or more surfaces from the boundary surface representation of architectural components (walls, slabs, etc.) are either incorrectly oriented i.e. the direction of the normal vector of the surface calculated using the right-hand rule is reversed (first type of surface error), or are missing from the boundary representation (second type of surface error). Examples of surface errors are displayed in figure 4. Part I of figure 4 displays a correctly oriented boundary representation of a building slab. In part II of figure 4, the first type of a surface error is illustrated.

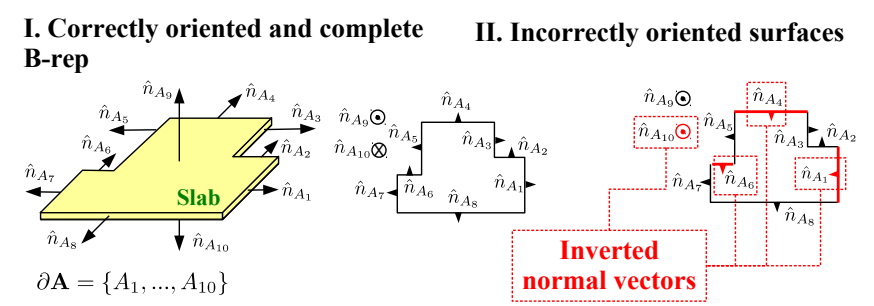

III. Incomplete B-rep

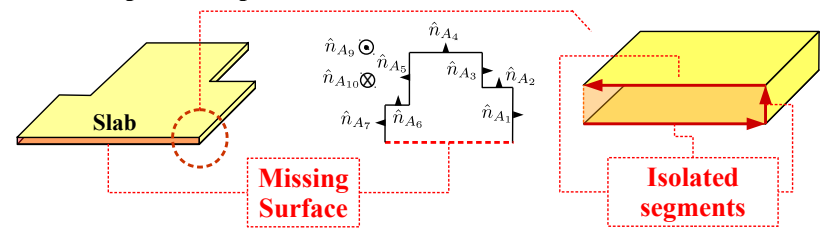

Figure 4: Examples of surface errors: (a) Surface orientation error, (b) Incomplete shell error (missing surface)

The boundary representation of the slab used in part I of the same figure, has some surface normals inverted. Finally in part III an example of the second type of a surface error is displayed, where the a boundary representation has a missing surface defined by a set of isolated segments (segments which belong to only one boundary surface polygon), on the boundary of this missing surface.

\subsubsection{Space definition errors}

Space definition errors occur when an internal building space volume is not surrounded completely by other architectural elements (walls. slabs, openings) and small gaps of undefined space volumes exist between the internal space and surrounding building architectural elements, as presented in part I of figure 5. In part II of figure 5 a correctly defined building space is presented, and for comparison an incorrectly defined space is displayed in part I of the same figure.

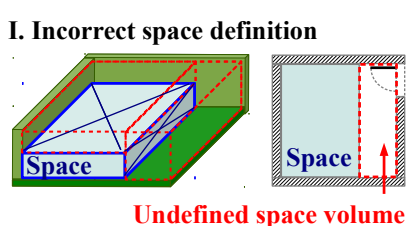

Space is incorrectly defined leaving undefined volume gaps indicated by the dashed lines

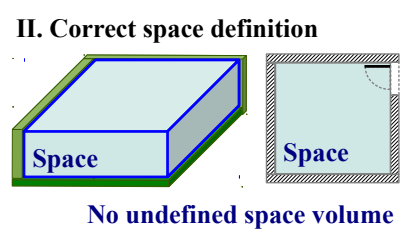

Space is correctly defined with no undefined volume gaps.
Figure 5: Examples of incorrect and correct space definition.

\subsubsection{Geometry Error Detection tool}

The Geometry Error Detection tool helps the modeler to generate a geometric error-free IFC BIM file by reporting any detected errors in in an XML form and exporting them in obj file format, for visualization purposes.

\section{Clash errors}

Clash errors are classified depending on the pair of involved entities. Several types such as Wall-Space, Slab-Space and others exist. Such classification is reported in by the GED tool as the following XML instances illustrate.

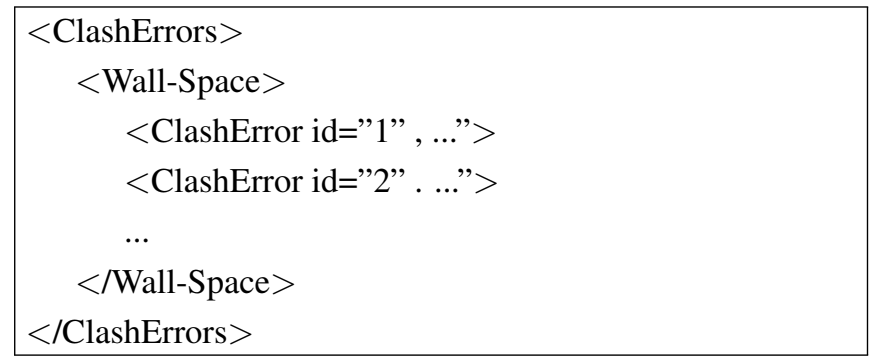

As displayed in the following XML instances, a clash error report contains the local building element ids (ID) and IFC global IDs (GID) of the two involved entities and the set of surfaces, which define the intersections among the two involved solid 
bodies.

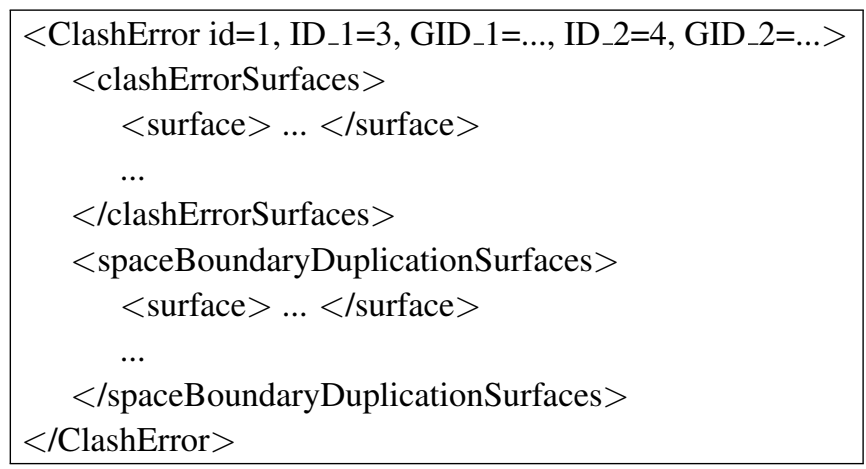

Every surface consists of an outer and possible multiple inner contours (holes) as described next:

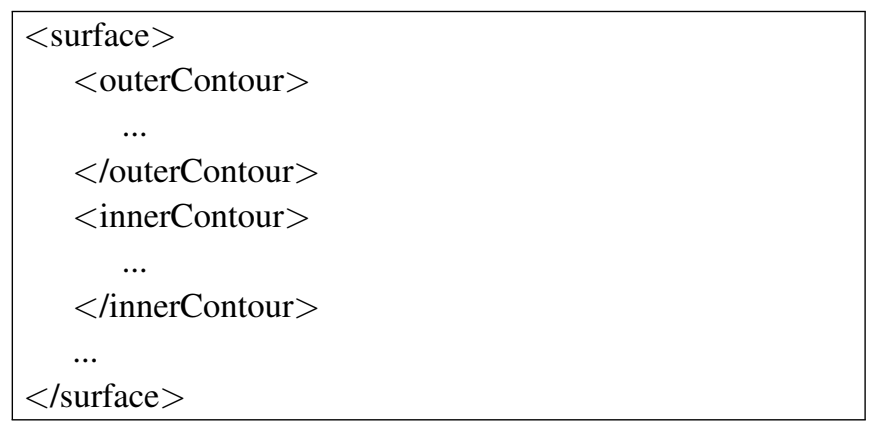

Finally the above outer and the inner contours are described by a set of points in three dimensions:

< point3D id="1" $\mathrm{x}=$ "19.0" $\mathrm{y}=$ ="3.2" $\mathrm{z}=$ "8.9"/>

$<$ point3D id="2" $\mathrm{x}=$ ="19.0" $\mathrm{y}=$ ="3.2" $\mathrm{z}=$ ="6.2"/>

...

Containment clash errors are also reported as regular clash errors with the word "containment" replaced by the word "clash" and with only the "spaceBoundaryDuplicationSurfaces" field.

\section{Surface errors}

Surface errors are linked to a solid body types, which can be either a wall, slab, space, e.t.c., and are reported by the GED tool as the following XML section indicates.

\begin{tabular}{|c|}
\hline$<$ SurfaceErrors $>$ \\
$<$ "Solid Body Type" $>$ \\
$<$ SurfaceError $\ldots>$ \\
$\ldots$ \\
$<$ SurfaceError $\ldots>>$ \\
$</$ "Solid Body Type" $>$ \\
$</$ SurfaceErrors $>$ \\
\hline
\end{tabular}

For each individual surface error GED tool reports the local ID of the related building entity, its IFC global ID (GID), the total surface norm of its boundary representation, the number of its boundary surfaces and a set of either isolated segments or inverted surfaces, according to the following XML in- stance:

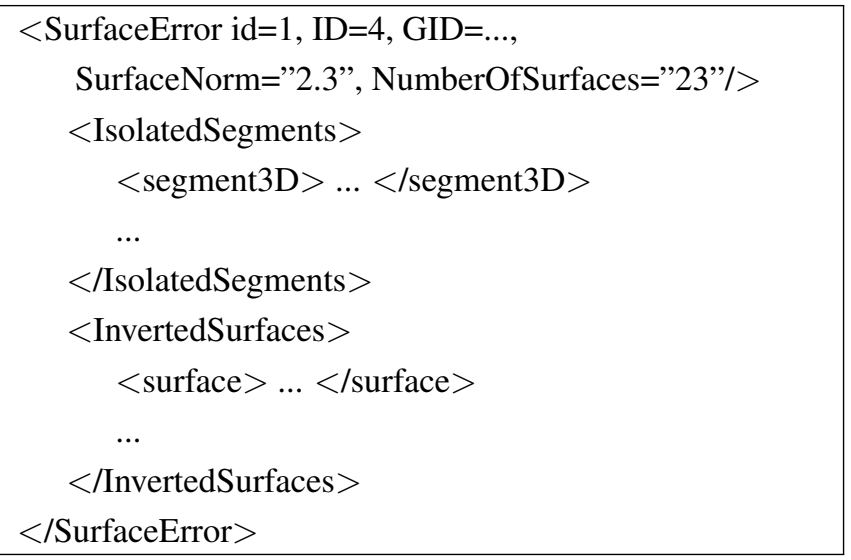

The inverted surfaces are surfaces which have incorrectly inverted normal vector and the isolated segments are line segments which belong to only one boundary representation surface and thus define a hole or a edge misalignment of the solid of the building entity. The inverted surfaces are defined by XML instances as regular surfaces as reported in the case of clash errors described previously, and the isolated segments are defined by the coordinate triplets of the initial $\left(x_{1}, y_{1}, z_{1}\right)$ and the final points $\left(x_{2}, y_{2}, z_{2}\right)$ of the segment as follows:

$<$ segment3D id="1", length=..., x1=.., y1, z1, x2, y2, z2/>

The total surface norm of the boundary representation of the solid body which has a surface error, is the Euclidean norm of the sum of the surface vectors of the boundary representation of the solid body. Any divergence of this surface norm from the zero value could be an indication of a surface error. The magnitude of the divergence of the surface norm from the zero value, is proportional to the severity of this surface error.

\section{Space errors}

Finally, a space definition error is reported from the GED tool, as displayed in the following XML instance:

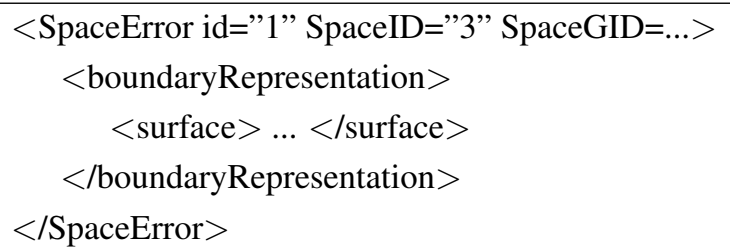

As it is presented in previous XML instance, for a space definition error the IFC global id of the internal space is given, together with the boundary surfaces of the space which are not attached to any other building architectural element. The space boundary surface is specified as polygon(s) with outer (and possible inner) 3D point contours. 


\section{EXAMPLES}

\subsection{Case study buildings}

The introduced BIM data quality checking tool is validated on two buildings: the Demo4 building which is an imaginary two-story office building, pictured in part I of figure 6 and the Turina Torre building located in Valladolid in Spain, displayed in part II of figure 6 .

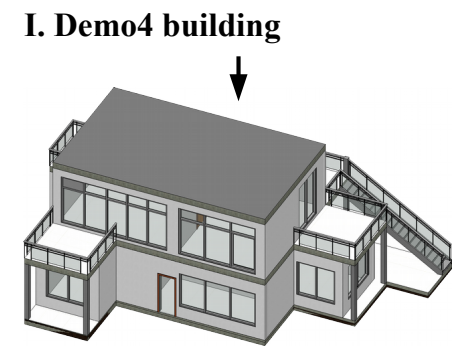

II. Turina Torre building

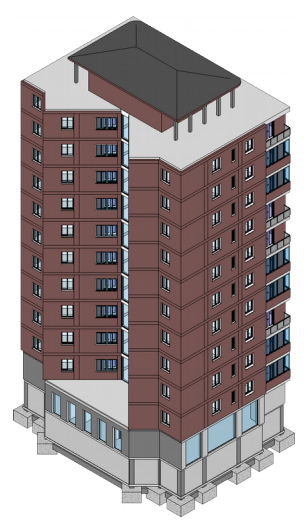

Figure 6: Case study buildings for the introduced BIM quality checking tool.

\subsection{Application of the BIM checking tool on Demo4 building}

The BIM Checking tool subcomponent is validated on Demo4 building and some error report message examples are presented next.

- The rule "space boundaries, parent space boundaries, corresponding space boundaries" has the following problems:

issue related to the MODEL, 2nd level space boundaries are missing.

- The rule "spaces, loads, parameters, schedules" has passed.

- The rule "doors, thermal parameters" has the following problem

issue related to Single-Flush:30" x 80" 2:245315 (1ChqrYs197G8E9uZmTikEW) "visual light transmittance" parameter is missing

- The rule "windows, thermal parameters" has the following problems:

issue related to M_Curtain Wall Awning:

M_Curtain Wall Awning:218240 (3RvIiUq4X8GweJx_3dFm2s) "visual light transmittance" parameter is missing ...
- The rule "plates, thermal parameters" has the following problems:

issue related to System Panel:Glazed:214779 (3RvIiUq4X8GweJx_3dFnBD) "visual light transmittance" parameter is missing ...

\subsection{Application of the GED tool on Turina Torre building}

The operation of the Geometric Error Detection tool subcomponent (GED tool) is demonstrated on the Turina building. As it is displayed in the MeshLab screen shots of the obj file exported by the GED tool, which are collected in figure 7 , several geometric errors were detected in the IFC file of the Turina Torre building. (space)-(slab opening) clashes (part I), wall opening containment clashes (part III), space errors caused by omitted opening volumes (part II) and surface errors cased either by normal inversion (part IVa) or isolated segments (part IVb), all affecting BEPS model generation, were detected.

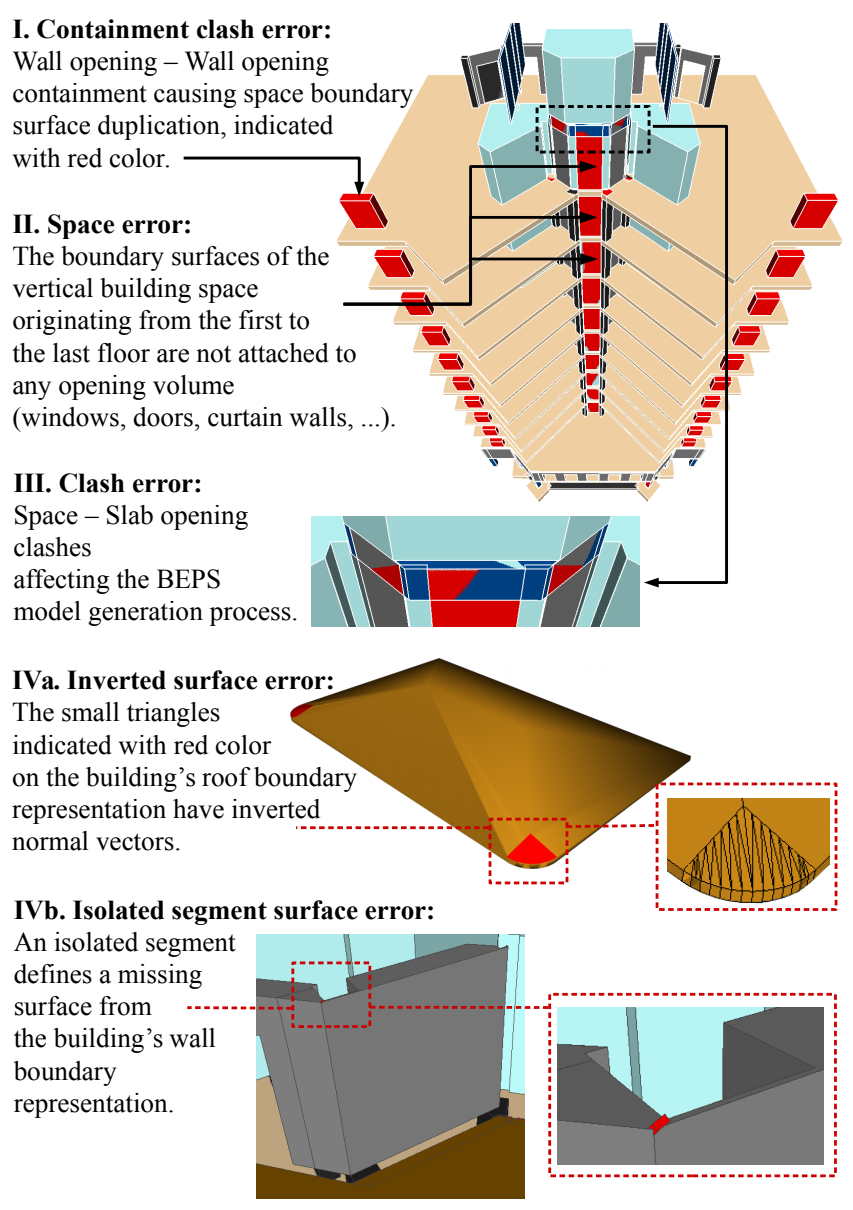

Figure 7: Demonstration of Geometric Error Detection tool's operation on Turina building (MeshLab screenshots of the obj file exported by the GED tool). 
A stand alone tool which performs BIM for BEPS suitability checking operations on IFC4 files was introduced. These operations are data quality checking operations which include: consistency checks (IFC4 schema compatibility check), completeness checks performed by the BIM Checking tool (space boundary, material and space related data checks) and correctness checks performed by the GED tool (detection of clash, space and surface geometric errors affecting BEPS model generation). To facilitate "BEPS-ready" BIM generation, a dedicated IFC exporter for Revit ${ }^{\mathrm{TM}}$ and appropriate BIM design guidelines are also provided to the user of the introduced tool.

The BIM for BEPS data quality checks performed by the introduced tool, should not be limited to building geometry, materials and operation schedules but should extend to other building components such as systems and their properties. Such investigation is a topic of ongoing research work. Additionally other error reporting formats such as the BCF file format (Stangeland, 2013), will be also included in the future.

Although multiple general BIM checking tools are available such as Solibri Model Checker ${ }^{\mathrm{TM}}$ (Solibri, 2018) and TEKLA's Model Checker Suite $^{\text {TM }}$ (TEKLA, 2014), the novelty of the introduced tool relays on the fact that it contains only the checking operations that will guarantee accurate BEPS model generation and, in this sense, is more specialized. Finally, it is important to mention that without the modeler's experience, even with the best checking tool available, the BIM for BEPS accuracy cannot be guaranteed.

\section{ACKNOWLEDGEMENTS}

Part of the work presented in this paper is based on research conducted within the project "Optimised Energy Efficient Design Platform for Refurbishment at District Level", which has received funding from the European Union Horizon 2020 Framework Programme (H2020/2014-2020) under grant agreement $\mathrm{n}^{\circ} 680676$.

\section{REFERENCES}

Alshehri1, F., Kenny, P., Pinheiro, S., and James, O. (2017). Model View Definition (MVD) for Thermal Comfort Simulation in Conventional BEPS tools. In PLEA, Edinburgh, UK.
Autodesk (2018). Revit Architecture. https://www. autodesk.com/products/revit/architecture.

Bazjanac, V. (2010). Space boundary requirements for modeling of building geometry for energy and other performance simulation. In $C I B W 78$, Cairo, Egypt.

Cormier, A., Robert, S., Roger, P., Stephan, L., and Wurtz, E. (2011). Towards a BIM-based service oriented platform: application to building energy performance simulation. In Building Simulation IBPSA Conference, pages 2309-2316, Sydney, Australia.

Gholami, E., Kiviniemi, A., and Sharples, S. (2015). Implementing Building Information Modelling (BIM) in Energy-Efficient Domestic Retrofit: Quality Checking of BIM Model. In CIB W78 conference.

Graphisoft (2018). Archicad 22 - BIM inside and out. http://www.graphisoft.com/archicad/.

Gudnason, G., Katranuschkov, P., Balaras, C., and Scherer, R. (2014). Framework for Interoperability of Information Resources in the Building Energy Simulation Domain. In Computing in Civil and Building Engineering, pages 617-624.

Hitchcock, R. J. and Wong, J. (2011). Transforming IFC architectural view BIMs for energy simulation: 2011. In Building Simulation conference of IBPSA.

ISO16739 (2013). Industry Foundation Classes (IFC) for data sharing in the construction and facility management industries. International Organization for Standardization (ISO).

Lilis, G. N., Giannakis, G., and Rovas, D. (2015). Detection and semi-automatic correction of geometric inaccuracies in IFC files. In Building Simulation IBPSA Conference, pages 2182-2189, Hyderabad, India.

Maile, T., ODonnell, J., Bazjanac, V., and Rose, C. (2013). BIM-Geometry modelling guidelines for building energy performance simulation. In Building Simulation IBPSA Conference, pages 3242-3249.

Nasyrov, V., Stratbücker, S., Ritter, F., Borrmann, A., Hua, S., and Lindauer, M. (2014). Building information models as input for building energy performance simulation-the current state of industrial implementations. In European Conference on Product and Process Modelling, pages 479-486.

Nemetschek Group (2018). Allplan. https://www.allplan. com/en/.

Pinheiro, S., Wimmer, R., ODonnell, J., Muhic, S., Bazjanac, V., Maile, T., Frisch, J., and van Treeck, C. (2018). MVD based information exchange between BIM and building energy performance simulation. $A u$ tomation in Construction, 90:91-103.

Senave, M. and Boeykens, S. (2015). Link between BIM and energy simulation. Building Information Modelling (BIM) in Design, Construction and Operations, 149:341-352.

Solibri (2018). Solibri Model Checker. https://www. solibri.com/products/solibri-model-checker/.

Stangeland, B. (2013). BIM Collaboration Format (BCF). BuildingSMART International User Group; BuildingSMART: Bakkmoen, Norway.

TEKLA (2014). Model Checker Suite. https://www. tekla.com/. 